\title{
DE LA WEB A LAS PLATAFORMAS EDUCATIVAS. ANÁlisis DE UNA EXPERIENCIA EN UN DEPARTAMENTO UNIVERSITARIO DE MATEMÁTICAS
}

\author{
(OF THE WEB TO THE EDUCATIONAL PLATFORMS. ANALYSIS OF AN EXPERIENCE IN A \\ UNIVERSITY DEPARTMENT OF MATHEMATICS)
}

Juan Duarte Vargas

Universidad de Antofagasta (Chile)

\section{RESUMEN}

La adaptación al uso de nuevas tecnologías cuando se va a enfrentar el proceso educativo en un ambiente moderno presenta por un lado el natural rechazo al cambio y por otro la ya consabida motivación a realizar el proceso reemplazando rápidamente lápiz y papel por medios tecnológicos cada vez más novedosos.

Este trabajo presentará la evolución que en forma natural y constante ha ido produciéndose tanto en profesores como en estudiantes del Departamento de Matemáticas de la Universidad de Antofagasta (Chile) al reemplazar el trabajo de aula por el trabajo combinado del espacio del aula con el de Internet. A través de la red el alumno puede acceder a un material preparado por nuestros profesores primero en Web educativas y posteriormente en plataforma educativa aumentando con este nuevo escenario los formatos de comunicación. Este es sólo el comienzo del trabajo dinámico y adaptativo que deberá hacerse permanente desde ahora en adelante.

Palabras clave: Web, plataforma educativa, proceso educativo.

\begin{abstract}
The adaptation to the use of new technologies when is going to face the educational process in a modern environment presents on the one hand the natural refusal to change and by another the already usual motivation to carry out the process replacing quickly pencil and role by technological media increasingly more novel.
\end{abstract}

This work will present the evolution that in constant and natural form has gone being produced so much in professors as in students of the Department of Math of the University of Antofagasta (Chile) 
to replace the work only of classroom by the work combined of the space of the classroom with that of Internet. Through the network the student can agree to a material prepared and placed by our professors first in Web educational and subsequently in educational platforms enlarging with this new setting the formats of communication. Of course, this is only the beginning of the adaptive and dynamic work that should be done permanently from now on.

Key words: Web, educational platform, educational process.

A fines de la década de los 80 y comienzo de los 90, en el siglo pasado, académicos del Departamento de Matemáticas de la Universidad de Antofagasta, Chile, retomábamos una iniciativa que habíamos comenzado en los años 70. En estos años iniciábamos la introducción del avance tecnológico en las tareas de aula. Se aprendía lenguajes de programación para producir material educativo aprovechando la posibilidad de efectuar cálculos muy rápidos y la visualización gráfica de lo que estaba ocurriendo con variables y sus relaciones.

A comienzos de década de los 90 ya estábamos vislumbrando un nuevo cambio, porque si bien es cierto que se estaba produciendo software matemático y estadístico que usaba los contenidos teóricos, producían una distracción en los estudiantes al tener que dedicar parte de su tiempo a aprender muchas palabras y sintaxis informáticas para producir los efectos deseados. Los informáticos advirtieron esto y fueron evitando que el usuario se viese involucrado en la programación misma.

Hacia fines de la década de los 90 Internet estaba ya masificado y preocupaba fuertemente el impacto que estaba produciendo en el estudiantado de todo nivel. Se convierte en una necesidad, entonces, la creación de páginas y sitios Web al servicio educativo pues la sociedad completa estaba cambiando sus valores, estaba teniendo nuevos intereses y había que reconcebir la educación masiva, abierta ahora a un nuevo ámbito.

El presente trabajo no pretende ser una exposición de las teorías en las que se sustentan las tendencias actuales, sino más bien cómo ha sido nuestra reacción ante la necesidad del cambio, buscando que las teorías se conviertan en una práctica confirmatoria. 


\section{TIC EN EL DEPARTAMENTO DE MATEMÁTICAS}

Nuestro Departamento de Matemáticas debatía reiteradamente sobre la importancia del impacto de las TIC, hasta decidir, como muchos otros antes que nosotros en el mundo, incursionan en este nuevo universo de trabajo (Duarte, J., 2004). Era necesario dar forma a un proyecto para lograr colocar el avance informático en TIC (cada vez más innovador) al servicio de la educación en matemáticas y estadística, que son las áreas de nuestra Unidad.

\section{Planificación del Proyecto}

Enfrentados al problema de planificar un plan para enfrentar la puesta en escena de un sistema educativo mediante la Red, se analizó diversos aspectos que se debía tener en cuenta:

- El software: Este era el elemento imperante en las investigaciones de matemática educativa. Sofisticadas calculadoras de bolsillo ya no eran sólo "calculadoras". Se comenzaba a producir algunas con librerías de aplicación matemática y estadística bastante atractivas con cantidades de datos aceptables para un análisis en el aula. Esta era una opción a la imposibilidad de tener ordenadores en una cantidad suficiente para la atención de todo el alumnado. Los ordenadores han sido, finalmente, la opción adecuada y en ellos el software.

En la línea de las ciencias, y particularmente de las matemáticas, la industria del software se esfuerza por ofrecer productos actualizados, de cada vez mayor espectro temático y más sofisticadas formas de presentación, acordes o no con la concepción educativa que el profesor quiere para su clase y aula presencial.

El profesor se ve enfrentado incluso a concebir el software como un elemento en el cual puede lograr nuevos conocimientos, pues éste se actualiza con los más trascendentes resultados de investigación y con las últimas líneas profesionales incorporadas al uso masivo en el mundo. El profesor siente la presión de actualizarse tanto en los nuevos conocimientos de su línea profesional, ofertados a modo de aplicación en los menús del software, como en el conocimiento y manejo de la última versión de éste.

La capacitación del profesorado, entonces, debe ir en la dirección del manejo experto de las ofertas del mercado del software y particularmente de la versión adquirida. 
La resistencia natural al cambio hace que los profesores analicemos una y otra vez el efecto que se está logrando con la introducción de estos elementos en la actividad docente. Se produce el natural enfrentamiento entre lo que habitualmente se hacía y el cambio que hoy nos impone el medio.

- Internet: Este es otro elemento en el cual el profesor actual debe incursionar, particularmente el profesor de ciencias matemáticas. La libertad del estudiante para navegar en la Red, le permite acceder a páginas de contenidos de diversa calidad, muchas de ellas escritas por autores no idóneos. En este sentido, al menos dos son las tareas que debe seguir el profesor:

- En primer lugar, inculcar en sus alumnos un espíritu crítico, logrando previamente en ellos una capacidad de razonamiento y de conocimientos suficientes como para que puedan efectuar discernimientos que le permitan evaluar el material que tienen ante sí. Debe observarse que este objetivo no es fácil de lograr pues se trata de personas en formación, con un espectro de conocimientos en aumento y porque en forma natural tienden a rendir culto a la imagen y a la forma (más que al fondo) del material que se les presenta. Forma y fondo deben ser analizados con claridad.

- En segundo lugar, complementando a lo anterior, el profesor debe seleccionar sitios interesantes que ayuden a concebir el objetivo de su clase. Con ello se verá privilegiada la actividad de aprendizaje en cuanto al tiempo utilizado en ella. Debe notarse que en muchos casos los sitios visitados por el alumno en una navegación de búsqueda actúan como distractores más que como reforzadores.

- Creación de Páginas Web: Advirtiéndose la facilidad con que la navegación en Internet capta la atención y el quehacer de los estudiantes, se comienzan las primeras incursiones en la creación de un sitio Web educativo del Departamento de Matemáticas. Se debía lograr que los académicos ya no centraran su actividad docente sólo en el trabajo de aula presencial, sino también en la creación de espacios virtuales que dotados de una buena organización y contenidos atractivos pudiesen evidenciar su objetivo educativo y a la vez ser atractivos para el estudiantado.

Lentamente el avance tecnológico y el uso de la Red hasta incorporarla al quehacer cotidiano obligan a ampliar y diversificar la tarea del profesorado. Cada vez más universidades y colegios van creando sus portales Web y van ampliando la forma de 
comunicación entre alumnos y profesores. Las nuevas cohortes que ingresan a las universidades traen formación en TIC. El profesorado debe cambiar y adaptarse al cambio.

Nuestro Departamento de Matemáticas se suma a las instituciones interesadas en el uso educativo de Internet. Logra, mediante un proyecto de mejoramiento de la calidad de la educación superior, MECESUP del Ministerio de Educación, la construcción de un pabellón de aulas informáticas destinado al trabajo en matemáticas y estadística con TIC (CRAMESTAP, Centro de Recursos de Aprendizaje en Matemática y Estadística Aplicadas). En 2002, establece una relación académica con el grupo de investigadores de Edulab ${ }^{1}$, de la Facultad de Educación de la Universidad de La Laguna, en Tenerife, España, liderado por el doctor Manuel Area Moreira y obtiene el apoyo necesario para dar un impulso de actualización en TIC a sus académicos matemáticos.

Es claro que la oferta de recursos iniciales para incursionar en la creación y mantenimiento de páginas Web no era muy motivadora, al menos en principio, para los matemáticos no dedicados a programación o a cosas informáticas. La dificultad para escribir símbolos matemáticos y fórmulas pasaba por transformarlas en imágenes para poderlas incorporar a un texto en HTML. El natural rechazo al cambio ante "tanta dificultad" estimulaba a unos a buscar soluciones ingeniosas y desanimaba a otros menos expertos en la materia. Pero para todos era claro que en algún momento había que incorporar estas habilidades y conocimientos al servicio de este nuevo frente educativo.

- Plataformas educativas: El siglo XXI traía también otro cambio en los valores globales. El desarrollo informático permite, en principio, la creación de plataformas para uso educativo que por sus costos no permite la atención masiva de usuarios mundiales. No obstante, interesados de todo el planeta se asocian para producir soluciones con el aporte de todos. Agrupaciones de personas interesadas en hacer masivo lo que a otros estaba dejando grandes ganancias, se unieron para aportar sus conocimientos y crean plataformas educativas de uso libre, entre ellas la que nuestro Departamento usa: Moodle ${ }^{2}$. El descubrimiento de las capacidades de Moodle nace de la continuas visitas a Edulab entre los años 2004 y 2006, lográndose en 2005 instalar esta plataforma para comenzar a trabajar en ella con los académicos más adelantados.

En este contexto, entonces, el plan inicial de perfeccionamiento académico en páginas Web ha sufrido un cambio a la par con el dinamismo de la innovación 
informática. Este es un cambio natural asumido plenamente por el cuerpo de profesores. En forma secuenciada desde 2001 académicos de nuestro Departamento visitaron centros mexicanos y brasileños, antes de obtener un contacto más cercano con Edulab, en España, para obtener actualización tanto en materias de tendencias educativas actuales como en TIC. Esto ha ido enriqueciendo nuestro quehacer gracias, en especial, al valioso y desinteresado apoyo tinerfeño.

El uso de Moodle ha permitido que muchos usuarios que no estaban interesados en tener que aprender tecnicismos, ahora pueden sólo limitarse a seleccionar la oferta (muy bien estudiada) de recursos incorporados para dar organización, sentido e intención a la labor educativa On Line.

Como parte de la planificación, nuestros profesores se están capacitando e incursionan en el uso de plataformas, debatiendo el potencial educativo disponible que hay que evaluar.

- Hardware: La existencia de plataformas nos planteaba la posibilidad de masificar aún más la labor educativa On Line. Ello conlleva la utilización de espacios lo suficientemente amplios para el almacenamiento de archivos de servidor y de software adecuado para trabajar en estos espacios virtuales. Un servidor con procesador y discos duros de velocidad y capacidad adecuada a la posible demanda de trabajo fue uno de los objetivos que logró hacer realidad nuestro Departamento.

\section{ESTRATEGIA}

Muchos análisis previos antes de llegar a la decisión de usar una plataforma educativa, nos permitieron dar un paso trascendental: tener nuestro propio espacio virtual dedicado a la docencia matemática y la estadística. Así lo entendieron las autoridades universitarias, cuyo apoyo fue importante, y también los académicos. En adelante ya no se trataba de ser experto creador de páginas Web sino de afianzar los diseños estructurales de la programación y de las actividades que el estudiante debería realizar en su visita a las páginas de cursos de la plataforma. Adquiría importancia el debate sobre la forma de comunicar, pues la calidad del contenido estaba garantizada.

La importancia y la cada vez mayor cantidad de usuarios de Moodle en el mundo, permite vislumbrar la posibilidad de intercambiar experiencias y opiniones para el 
mejor uso y mejora de esta plataforma. Nos dábamos cuenta de que sus habilidades iban en aumento y esto nos ayudaba a motivar a nuestros profesores a colocar sus cursos en la Red. Era indudablemente atractivo que Moodle fuese, además, una plataforma amistosa, por lo que al ser nosotros usuarios no informáticos, a muchos producía un encanto especial el dedicar más tiempo a la comunicación con el alumno por una cantidad mayor de vías (aula, tutorías, plataforma, e-mail,...) con el fin de lograr el aprendizaje matemático y estadístico, en lugar de la creación, diseño y mantenimiento de sitios educativos más bien "personales".

\section{IMPLANTACIÓN DEL PROYECTO}

CRAMESTAP está dotado de un Laboratorio Tecnológico, 30 estudiantes frente al ordenador, con espacio para proyector y conexión a Internet; un Laboratorio Multifuncional, 20 alumnos frente al ordenador dispuestos en filas laterales, que dejan al centro un espacio para 25 mesas funcionales que dispuestas para el trabajo en grupo antes de acceder al ordenador (posee también conexión a Internet y proyector para las disertaciones); un par de pequeñas salas, en un ambiente aislado y de concentración: sala de Recursos Bibliográficos y sala de Recursos Docentes, en las que se ha colocado a disposición de los académicos material bibliográfico de matemática, estadística y tutoriales de software disponible acorde con los cursos que se están ofreciendo, dotadas de otros cuatro ordenadores para la creación, prueba y discusión del material que se colocará en la plataforma; finalmente, tiene una sala de Administración en la que se aloja el servidor que alberga a la plataforma.

Obviamente, todo este equipamiento está supervisado y mantenido por un Administrador que se ocupa de los aspectos informáticos y de la puesta en funcionamiento permanente de equipos y software. La labor del Administrador es crucial para el funcionamiento de las actividades docentes de aula y On Line, además del cuidado, atención y actualización de la plataforma, como también de la inspección del trabajo de los usuarios.

El cambio de escenario desde Web a plataforma, hace necesaria la capacitación constante del profesorado sobre las últimas novedades tanto de la plataforma como del software matemático y estadístico disponible. El trabajo con simbología matemática presenta a nuestros académicos un particular desafío en el uso de plataforma. Se salva la situación usando Word y transformando a PDF. 


\section{FOCALIZACIÓN DE LAS ACTIVIDADES}

Se ha vislumbrado dos etapas en la puesta de material y actividades educativos en Moodle, a la vez de estar preparados para la solución de problemas que se presenten:

\section{Etapa I: Capturar y mantener el interés de los académicos}

Por supuesto, el objetivo de la Dirección de Departamento es tener en la plataforma todos los cursos que se dictan a las distintas Facultades de la Universidad, pero es estimulante tener como primer objetivo de trabajo la mayor cantidad posible, buscando lograr la experiencia necesaria en los académicos y la confianza de que el tiempo invertido se recupera después al tener un interesante acopio de material del que se puede disponer posteriormente en cualquier momento para su actualización, rediseño o impresión.

Cuando hay varios grupos dentro de un mismo curso, cada uno a cargo de un profesor posiblemente distinto, Moodle permite definir Coordinador de Curso al que liderará el trabajo de estos académicos para ofrecer a los estudiantes un mismo material, actividades, recursos y tareas, de manera que el factor profesor, en estos casos, quede reducido a su efecto solamente en la presentación y estimulación del alumno en el aula frente a un temario estandarizado. Un trabajo asociado por parte de los profesores permite que interactúen en beneficio del diseño, material, actividades y tareas dispuestos para el alumnado, además de no aislar innecesariamente, en principio, a aquellos académicos que necesitan experiencia antes de realizar trabajos On Line.

\section{Etapa II : Dar a cada curso el máximo sentido educativo}

Una vez que el material de lectura y de la clase de aula está preparada y dispuesta en Moodle (esta primera experiencia puede durar un semestre o más para no producir rechazo natural de los profesores) se procede a completar las actividades de curso en cuanto a cuestionarios dirigidos y evaluaciones en línea que realiza el estudiante por sí mismo para medir su estado de aprovechamiento. Los cuestionarios dirigidos pretenden reemplazar a los ejercicios ejemplo de la clase tradicional. Éstos en muchos casos eliminan el interés de algunos alumnos en ser guiados o evaluados por el profesor mientras realizan su propuesta de solución. Se trata de que el profesor identifique esta vez los pasos que ha de seguir el alumno para solucionar el problema y entregarlos a modo de preguntas. De esta manera el estudiante estará realizando la 
solución guiado por el cuestionario dispuesto por el profesor. Esta "guía" permite al alumno "descubrir" el procedimiento a seguir en casos similares. La mayor parte de nuestros académicos se encuentra en un ambiente positivo y motivador que augura un cambio a mediano plazo.

\section{El enfrentamiento de problemas informáticos:}

El trabajo inicial en páginas Web, aparte del diseño de la página misma y del sitio que la albergaría, no gozaba de mucha aceptación por parte del profesorado. Esto porque la simbología matemática no era fácil de trabajar en HTML. Para muchos no resultaba atractivo. La única ventaja visible era que este material estaba dispuesto a toda hora para el estudiante donde quiera que este se encontrara y tuviera a su alcance ordenador y conexión a Internet. Para muchos esto era poco, educativamente hablando. Algunos tratábamos de crear páginas educativas con mucho ingenio, tratando de captar la atención del estudiante. Otros intentaban con Power Point, Flash, Director o, los más interesados, con Java, entre otras formas disponibles. Pero en todo caso, siempre lo que se pudiera utilizar pasaba por una capacitación y perfeccionamiento en software y lenguaje necesarios.

El disponer de una plataforma era un gran paso. El haber optado por Moodle otro. Pero este último no estaba exento de algunas dificultades que hemos ido salvando. En primer lugar parte del profesorado deseaba escribir directamente en los campos de Moodle y no tenía a disposición la posibilidad de crear sus fórmulas allí. Los que poseían conocimientos de LaTex podían aprovechar este lenguaje para escribirlas. Pero esto no estaba al alcance de todos. Prácticamente nadie estaba inclinado a usar esta posibilidad.

Parte de las presentaciones iniciales se realizaron con $\mathrm{eXe}^{3}$ mejorando con esto la apariencia de los temas. eXe cuenta con una estructura que permite agregar cuestionarios para medir el aprovechamiento del estudiante, lecturas recomendadas, consejos, actividades, casos, definiciones, diagnósticos, discusiones, glosario, diario, experimentos, multimedia, reflexiones, revisiones, resúmenes, enlaces, etc. Esto permitía crear pequeñas dosis de temario en los que el estudiante iba recibiendo consejos, tareas y reflexiones para su análisis.

Paralelamente el uso de Hot Potatoes ${ }^{4}$ también ayudaba al trabajo interactivo de presentaciones educativas. Resultaban habilidades interesantes de Hot Potatoes: JQuiz, para realizar cuestionarios y dando puntajes a cada respuesta; JCloze, para completar texto escribiendo la palabra que falta; JCross, para hacer crucigramas con 
puntaje para cada respuesta; y JMatch, para emparejar las opciones correctas. Los puntajes van disminuyendo en la medida en que se recurre a ayudas. Algunas de estas habilidades están disponibles en la plataforma Moodle como Hot Pot.

La dificultad para crear archivos de poco peso o para custodiar la autoría hacía que nuestros académicos usaran las bondades ofrecidas por Adobe ${ }^{5}$ para la creación de documentos PDF. Es más, se dispuso de opciones free para transformar documentos a formato PDF (PDF $995^{6}$ ). Todo esto constituyó permanente diálogo entre los académicos para instalar y usar estos utilitarios favoreciendo el ambiente para la creación de material educativo de los cursos.

Como si fuera poco, últimamente los que estaban agobiados por no poder colocar fórmulas matemáticas en los campos de Moodle, por no saber LaTex, ahora disponen de una versión de Texaide ${ }^{7}$, que es un émulo del editor de ecuaciones ofrecido por los creadores de Math Type ${ }^{8}$ para transformar las fórmulas matemáticas a LaTex simplemente escogiendo en una ventana semejante a la del editor de ecuaciones de MS Word los símbolos que se desea escribir. Este software permite que cuando la fórmula se copia y se pega en el campo de escritura de páginas en Moodle, queda automáticamente escrita en LaTex. Cuando se guarda el recurso y luego se pide verlo, la escritura de la fórmula es como se deseaba. Esto agradó a muchos matemáticos y estadísticos.

Si se analiza el funcionamiento de la plataforma en el servidor, se puede decir que gracias a que se ha debatido todos los aspectos que pudieran ser de interés, se ha estado en condiciones de enfrentar sin problemas la puesta en marcha, uso y mantenimiento, tanto de equipos de aula como de servicio de parte del servidor durante las 24 horas.

El problema es ahora que la demanda por el uso de las aulas de CRAMESTAP ha aumentado y eso nos parece muy bien para el logro de los objetivos trazados. Pero a la vez nos obliga a pensar en una ampliación de los recintos para aumentar la capacidad de atención de la demanda de los estudiantes tanto como de los profesores.

\section{LA GESTIÓN}

Evidentemente el aspecto más importante en todo este proceso que está viviendo el Departamento de Matemáticas se debe a la gestión realizada por la Dirección de Departamento y a las directrices que se están dando desde el gobierno universitario 
y también del país. La gestión realizada desde fines de los años 80 ha pasado por varias etapas:

\section{Aulas de trabajo docente teórico y práctico}

Uno de nuestros primeros objetivos fue lograr un espacio físico en el cual reunir la actividad de nuestro Departamento, particularmente para la utilización de elementos informáticos en beneficio del trabajo docente. Las autoridades universitarias daban todo su apoyo a proyectos innovadores entre los cuales se encontraba el nuestro. Las directrices del Ministerio de Educación estimulaban hacia las TIC. La Facultad de Ciencias Básicas, de la cual depende nuestro Departamento, coincide con nuestras aspiraciones y postula un proyecto conjunto reuniendo al nuestro con los de los Departamentos de Física y Química, también dependientes de ella. Se postula, entonces, con este proyecto conjunto al llamado a concurso de proyectos MECESUP realizado por el Ministerio de Educación de Chile a comienzos de la década de los 90. El ganar este proyecto dio vida a lo que es hoy CRAMESTAP. El espacio físico estaba logrado.

A las aulas usuales de trabajo teórico se agregaban ahora las de trabajo con elementos informáticos en el Laboratorio Multifuncional y en el Laboratorio Tecnológico. Los docentes cuentan ahora con ordenadores y un espacio dispuestos especialmente para la discusión, análisis y creación de material de apoyo a las actividades docentes.

\section{Servicio de Administración}

Se gestionó también el servicio permanente de un profesional informático Administrador de CRAMESTAP. Esto permitió obtener una organización de la gestión de aulas e Internet, estructurando la atención mediante dominios que permiten el funcionamiento eficiente conforme a los privilegios que necesitan los usuarios para su uso específico. La principal preocupación del Administrador es el funcionamiento continuo y óptimo tanto de los equipos para la atención de académicos y estudiantes en su trabajo presencial como del permanente servicio que debe prestar el equipo servidor para el trabajo en línea de nuestros académicos y estudiantes desde cualquier punto geográfico. Debe decirse que en nuestra región desértica, el estudiantado proviene en algunos casos de localidades que distan 200 o más kilómetros. 


\section{Adquisición y renovación de licencias de software:}

Parte de la gestión es también la adquisición y renovación de licencias de software estadístico y matemático, informático o utilitario. Esto permite que nuestros proveedores nos mantengan actualizados y recibamos ofertas también de otros productores.

\section{Mantención o renovación de equipos:}

Una buena gestión de CRAMESTAP exige contemplar la mantención y renovación de equipos: ordenadores, proyectores multimedia, cámaras y webcams, climatizadores, mouse, etc. El avance vertiginoso de la tecnología ha exigido ya que se analice un proyecto de inversión institucional en renovación de equipos. Los tratados de libre comercio que está realizando el gobierno de Chile permiten esperar que lleguen a nuestro país una gama de productos informáticos variada con precios que permitan renovación y diversificación más frecuente.

\section{Capacitación académica}

Este es uno de los puntos más importantes. El uso de las TIC como una necesidad por parte de los académicos tiene como razón de ser la incursión en un medio por el que el estudiante está siendo capturado más y más. Las nuevas tecnologías llenan su atención y su quehacer. Ipods, MP3, MP4, Pen Drives, Internet, juegos, etc. encantan sus sentidos y le limitan sólo a reaccionar perdiendo casi el dominio y su potencial desarrollo mental.

La incursión académica usando estos medios es casi nula y educacionalmente, en este sentido, se pierde terreno a diario. La reacción de los académicos puede y debe generar un despertar al uso juicioso de estos elementos tornándolos un bien al servicio del desarrollo del intelecto y no sólo del deleite de los sentidos. Su uso mercantil con fines de lucro está ganando terreno al uso educacional al servicio de un modelo social adecuado y deseado por y para todos. El "nosotros" está cediendo terreno al "yo" y hasta en las aulas se observa la falta de comunicación entre las personas. Manejadores de la nueva tecnología disponen sus creaciones a usuarios inexpertos e influenciables fascinados por las estimulaciones que están teniendo sus sentidos. Colores, formas, movimientos, sonidos, secuencias, en fin, producen un efecto conquistador. 
La academia, para recuperar terreno, debe perfeccionar sus formas de comunicación con la finalidad de re-encantar a sus estudiantes. Es claro que se está en un mundo donde todo se sucede vertiginosamente. Podemos decir que más comunica un diseñador publicitario en un minuto, que lo que comunica un profesor de matemáticas en quince minutos de su discurso. Es parte de la razón por la que hemos perdido la capacidad de desarrollar intelectualmente a nuestros estudiantes. Si se analiza bien, no basta querer usar los elementos informáticos con fines educativos. Es necesario un buen perfeccionemos en esta tecnología para enfrentar cuanto antes este nuevo desafío que ha comenzado mucho antes de que nos diésemos cuenta. Tendremos que ser un poco publicistas, un poco diseñadores, pedagogos y muy conocedores de nuestra disciplina para sacarle partido en beneficio del sentido educativo que queremos lograr.

En este contexto, nuestro Departamento ha estado invitando a especialistas de diversas disciplinas para recibir información y perfeccionamiento en tendencias educacionales, desarrollo de presentaciones en software útil al sentido educativo que se busca, y participando, además en seminarios que permiten debatir sobre los lineamientos de gobierno en materia de educación, particularmente en lo que dice relación con acreditación.

Nuestra Facultad, además, es la primera en Chile que obtiene una certificación de calidad en norma ISO 9001 y NCH 2728 (norma chilena). Esto hace que nuestro quehacer se vea ajustado a inspección permanente.

\section{Alianza con otras universidades}

Nuestra Facultad de Ciencias Básicas está en conversaciones para establecer un convenio de cooperación con la Facultad de Educación de la Universidad de La Laguna. Se trata de compartir experiencias y desarrollar proyectos conjuntos, de manera de estudiar el comportamiento de las variables educacionales de ambas latitudes, en particular para nosotros las relacionadas con didáctica, comunicación y aprendizaje matemáticos.

\section{Organización administrativa para la ejecución del proyecto}

Cuatro son los cargos de Departamento relacionados directamenteconla ejecución de este proyecto con TIC: Director de Departamento (quien lo lidera), Secretario de Docencia y Perfeccionamiento Académico, Encargado de Inventario (que se responsabiliza del control y existencia de equipos, software y material bibliográfico) 
y el Administrador de CRAMESTAP. El funcionamiento mancomunado de estas cuatro personas permite que junto al Concejo de Departamento formado además por los Jefes de las Áreas de Matemáticas y Estadística, el Secretario de Investigación y el de Extensión, se analicen las situaciones que ameritan una decisión.

\section{El material didáctico disponible para el alumno}

La Vicerrectoría Académica de la Universidad convoca anualmente a la postulación de Proyectos de Docencia cuya finalidad es producir acciones de apoyo al quehacer docente de las unidades. Nuestro Departamento ha logrado producir, mediante estos proyectos, material de texto en teoría y ejercicios, además de material docente disponible en $\mathrm{CD}$.

Se suma ahora a esta oferta la puesta On Line de la temática desarrollada en forma clara y detallada por el profesorado en sus cursos.

La forma adecuada en que Moodle oferta sus recursos y actividades docentes está permitiendo a los profesores un nuevo frente de estructuración de su quehacer mostrando la organización de su trabajo docente del semestre. Con esto hace un público y transparente compromiso con sus alumnos y puede someter a evaluación constante el desarrollo de lo planificado.

La declaración de contenidos y su desarrollo, expuesto en forma de trabajo por semanas o por temas, permite al alumno tener una clara visión de la extensión y profundidad de lo que debe aprender. Puede incluso ganar tiempo preparando de antemano los temas que el profesor tratará en el aula.

El profesor puede, también, inspeccionar los ingresos y desplazamientos de sus alumnos por el curso y asistirles en caso de que sea necesario. Puede averiguar, de paso, por qué algunos se han alejado de las visitas a la plataforma haciendo un seguimiento a lo que tal vez pueda ser deserción. Se agiliza, así, el control de su actividad.

\section{Búsqueda, análisis, evaluación y determinación de los recursos de apoyo al trabajo con la plataforma: soporte técnico}

Este aspecto será responsabilidad de todos los profesores participantes en la formación de los estudiantes en el curso. Los recursos de apoyo pueden ser páginas interesantes encontradas en Internet, software gratis de acceso fácil para el 
estudiante o que pueda disponerse en la plataforma para ser instalado y trabajado por los alumnos, o bien utilitarios que beneficien la labor del estudiante en el curso (enlaces a Adobe Acrobat Reader para PDF; Winzip para descomprimir archivos; Hot Potatoes para variar la oferta de trabajo en ejercicios; eXe, para la preparación de temas en otra forma visual, en fin).

En caso de haber dificultades el Administrador de CRAMESTAP aconsejará sobre las soluciones.

\section{Integración del alumnado a la plataforma: Formas de acceder y utilizar el servicio docente que se ofrece en aula y On Line}

Se distribuyó un manual de uso para matriculaciones en cursos de Moodle a partir del cual los alumnos ingresan a los cursos a trabajar con el material preparado y dispuesto allí por sus profesores: desarrollo de contenidos en archivos pdf, presentaciones en power point, listado de ejercicios, consejos de trabajo para mejorar el aprendizaje, videos, foros e información general sobre la programación de actividades. Los alumnos pueden acceder en cualquier momento a todo este material en línea. Se entregan y reciben tareas con fecha de plazo perentorio y opcionalmente con un segundo plazo para mejoramientos de lo entregado.

La sesión de aula permite que el alumno reciba instrucción en la forma tradicional en algunos casos, en trabajo en grupos, con presentación y discusión de situaciones problemáticas y ejercicios prácticos. Puede usar software matemático y estadístico para apoyar su aprendizaje.

Se realizan dos tipos de evaluaciones. Los cuestionarios On Line de Moodle y las evaluaciones en aula por parte de un control escrito individual para cada alumno.

Toda la información que el estudiante necesita para su curso se realiza también a través de paneles, central de apuntes y en el curso dispuesto en Moodle.

Los cursos de Moodle son sencillos de navegar no es necesaria una capacitación profunda del estudiantado. 


\section{CONCLUSIONES}

Cuando se ha visto el camino recorrido con el detalle que aquí se ha hecho, realizando un análisis de lo actual, no se puede menos que estar satisfecho de lo logrado y seguir adelante sin mezquinar esfuerzos. El alumnado lo agradecerá. Debemos estar concientes de que mientras más claros y seguros vayamos por esta senda y más cuidado pongamos en quitar importancia a lo superfluo o intrascendente existente en la Red, mayor espacio estaremos ganando en beneficio de la formación del estudiantado.

Podemos decir, finalmente, que hemos logrado observar:

\section{Resultados en los estudiantes:}

- No existen mejoras sustanciales o cambios significativos en los resultados académicos de los estudiantes. Pruebas estadísticas adecuadas no muestran que haya cambio significativo en los rendimientos estudiantiles de antes de aplicar este proyecto y lo logrado posteriormente.

- Se observa, eso sí, una mejora notable en la actitud de los estudiantes al evidenciar más participación y motivación por las actividades académicas preparadas por sus profesores. El interés por el tratamiento de contenidos es diferente pues tienen más participación activa: resuelven, navegan, observan, comentan libremente, tratan de fundamentar, defender, probar, deducir adecuadamente.

- La relación profesor-alumno se ve beneficiada a través de e-mails y foros, produciéndose una mayor comunión de intereses e intercambio de ideas.

- Muestran sus "descubrimientos" y lecturas en la Red. Comparan con lo tratado en el aula. Proponen innovaciones en términos de "nuevos" elementos encontrados en la Red y que podrían ser un aporte al tratamiento de los temas matemáticos y estadísticos.

- Se interesan en el aprendizaje de software.

- Un porcentaje significativo muestra sus logros evidenciando un cambio de autoestima usualmente baja a expectante positiva (con satisfacción contenida hasta afianzar su logro). 
- Un porcentaje significativo, evidencia mayor claridad en la conceptualización (el computador exige respuestas correctas en los cuestionarios, no hay términos medios, lo cual aumenta de alguna manera la exigencia).

- Se observa una mejora en la lectura e interpretación de situaciones problemáticas.

- En el trabajo de aula, se observa un mayor interés por resolver problemas matemáticos y estadísticos, discutiéndose con mayor interés las situaciones en que la intuición se ve atacada por la evidencia práctica dada por gráficas o resultados de cálculos e indicadores computacionales.

\section{Resultados en los profesores:}

- Natural rechazo inicial pues al iniciar una metodología con nuevas tecnologías, el profesorado debe dedicar mucho tiempo a la elaboración previa de materiales.

- Cuando el profesor obtiene experiencia en la metodología con nuevas tecnologías siente que su trabajo disminuye con respecto a la elaboración de materiales y preparación de clases pues las posibilidades del "copiar", "pegar" y diseñar una reorientación y completación adecuada de lo ya trabajado, permite, en muchos casos, hacer aplicaciones similares de la matemática y de la estadística en campos diversos. Esto puede constituir una ventaja.

- La tutoría constituye un espacio importante en la tarea de enseñanza On Line. $\mathrm{Al}$ existir mayor interacción y participación de los estudiantes a través de nuevos canales de comunicación como pueden ser el correo electrónico o foros, el profesorado debe dedicar más tiempo a la resolución de dudas.

- El profesorado tiene claro que, al menos por ahora y mientras no se investigue más en la preparación de material autosuficiente para el aprendizaje personal, nada cambia la inmensa importancia de la comunicación directa entre seres humanos, en este caso profesor y estudiante, para la transmisión "no industrializada" de los valores sociales, teniendo en cuenta, además, que el sentir humano difícilmente se puede transmitir mediante una sesión On Line. Transmitir conocimientos por la Red parece ser más sencillo que transmitir valores sociales. Los profesores deberían ser todos buenos diseñadores gráficos y publicitarios. Eso aún no se ha discutido con amplitud de visión. La riqueza de un contacto presencial es invaluable y además necesaria. Es posible que algún 
día los sentidos de los seres humanos perciban a sus pares sin el contacto directo pero por ahora el cambio no se ha producido.

- El profesor deberá aprender de los cambios imperantes en el medio para incorporarlos adecuadamente a sus frentes de comunicación y para producir aprendizaje en su disciplina, particularmente si se trata de aprender y transmitir ciencia. El profesorado deberá concebirse con una profunda formación dinámica, dispuesta al cambio ágil para rápidamente aprender, desarrollar y aplicar, y también guardando consigo lo trascendental enfrentar el siguiente cambio que se acerca raudo.

- Darnos cuenta de que las "dificultades" que se presentan, particularmente, al profesor de matemáticas en estos tiempos de cambio son sólo el comienzo de un gran desafío que significa usar cuanto antes los elementos disponibles para comprender la observable falta de capacidad de abstracción (u oculta tal vez) de la gran masa del estudiantado. Esto está provocando la existencia de élites que acaparan el conocimiento y la información, dejando sólo para unos pocos la capacidad de formación para aprender.

- Nuestro Departamento ya ha tomado conciencia de su valioso inicio en TIC y debe comprometerse a custodiar los valores superiores de la educación en la era cibernética.

\section{Nuevas hipótesis para posteriores investigaciones:}

- Un hecho interesante está aflorando al observar un aumento del número de estudiantes que llegan a las conclusiones certeras en la resolución de problemas matemáticos, aun cuando no son conscientes de cómo llegan a obtener sus resultados. En principio se podría entender que llegan a los resultados correctos de manera fortuita, pero resulta, al menos curioso, que aumente el número de casos y que todos ellos tengan experiencia en el uso de ordenadores y videojuegos.

- Me parece interesante estudiar la relación del desarrollo cognitivo logrado como resultado del uso de ordenadores, Internet y videojuegos, con el proceso cognitivo necesario para el aprendizaje de las matemáticas, analizando en qué medida, esta relación es real y positiva. Los profesores de matemáticas deberíamos centrarnos en conseguir un desarrollo metacognitivo del estudiante con respecto a los procesos matemáticos, en lugar de efectuar en forma tal vez mecánica 
tanta ejercitación práctica que sólo condiciona. Es decir, debemos trabajar para que el alumno sea consciente del desarrollo que su mente lleva a cabo para la resolución de problemas y el aprendizaje matemáticos. Debemos trabajar para las estructuras y relaciones metacognitivas que desarrollen la capacidad de aprender y lograremos mayor y mejor aprendizaje de las matemáticas, en particular.

Una lectura interesante es la del siguiente link, en que se habla de logros con videojuegos, a los cuales pondría apellido: videojuegos educativos, que es lo que nos interesa (http://bibliobytes.unimet.edu.ve/Numero4/trabajo-grado.htm).

\section{NOTAS}

$1 \quad$ http://www.edulab.ull.es/index.htm

2 http://moodle.org/

3 eXe, eLearning XHTML editor, http://www.exelearning.org/.

4 http://hotpot.uvic.ca/

5 http://www.adobe.com/products/acrobat/

6 http://site4.pdf995.com/download.html

7 http://www.dessci.com/en/products/texaide/

8 http://www.dessci.com/en/

\section{REFERENCIAS BIBLIOGRÁFICAS}

Area Moreira, M. (2004). Los medios y las tecnologías en la educación. Madrid: Ediciones Pirámide.

Barberá, E.; Badia, A. (2004). Educar con aulas virtuales. Orientación para la innovación en el proceso de enseñanza y aprendizaje. Madrid: A. Machado Libros S.A.

Behar Gutiérrez, R.; Pere Grima, C. (2001). Mil y una dimensiones del aprendizaje de la estadística. Estadística Española, 43 (148), 2001, 189 - 207.

Cebrián de la Cerna, M. (Coord.) (2005). Tecnologías de la información $y$ comunicación para la formación de docentes. Madrid: Ediciones Pirámide.
Duarte Vargas, J. (2004). Del libro de texto al ordenador. Reconstrucción de una experiencia de docencia universitaria en matemáticas. Qurriculum Universidad de La Laguna, 17, octubre 2004, 237 -255 .

Luengo García, M. A. (2001). Formación didáctica para profesores de matemáticas. Madrid: Editorial CCS. 


\section{PERFIL ACADÉMICO Y PROFESIONAL DEL AUTOR}

Juan Duarte Vargas es Profesor de Matemáticas y Física, título obtenido en la Universidad Católica del Norte, en Antofagasta, Chile; Master en Estadística Matemática, grado obtenido en el Centro Interamericano de Enseñanza de la Estadística (CIENES), dependiente de la Organización de Estados Americanos en convenio con la Universidad de Chile; y Doctor en Ciencias Matemáticas, grado obtenido en la Universidad Complutense de Madrid, España. Coordinador del Programa de Magíster en Ciencias en la mención Estadística Industrial y Director del Departamento de Matemáticas de la Universidad de Antofagasta desde 2005.

E-mail: jduarte@uantof.cl

DIRECCIÓN DEL AUTOR

Departamento de Matemáticas

Facultad de Ciencias Básicas

Universidad de Antofagasta

Avenida Angamos 601

Antofagasta, Chile.

Fechas de recepción del artículo: 17/10/07

Fechas aceptación del artículo: 10/01/08 